\title{
TRATAMENTO CIRÚRGICO DE SIALOLITO DE GRANDES PROPORÇÕES EM GLÂNDULA SUBMANDIBULAR: RELATO DE CASO
}

\section{Fernanda Britto de Melo Silva}

Residente de Cirurgia Oral e Maxilofacial do Hospital Federal dos Servidores do Estado do Rio de Janeiro - HFSE e da Universidade Federal Fluminense - UFF, Niterói / RJ, Brasil.

\section{Natália Santos Carneiro}

Residente de Cirurgia Oral e Maxilofacial do Hospital Federal dos Servidores do Estado do Rio de Janeiro - HFSE e da Universidade Federal Fluminense - UFF, Niterói / RJ, Brasil.

\section{Eugênio Rodrigues Arantes}

Residente de Cirurgia Oral e Maxilofacial do Hospital Federal dos Servidores do Estado do Rio de Janeiro - HFSE e da Universidade Federal Fluminense - UFF, Niterói / RJ, Brasil.

\section{Rafael Seabra Louro}

Professor da disciplina de Cirurgia Oral Menor da Universidade Federal Fluminense UFF, Niterói / RJ, Brasil.

\section{Rodrigo Figueiredo de Brito Resende}

Professor da disciplina de Cirurgia Oral Menor da Universidade Federal Fluminense UFF, Niterói / RJ, Brasil.

Instituição na qual o trabalho foi realizado: Faculdade de Odontologia da Universidade Federal Fluminense - Niterói/RJ

Categoria: Relato de caso

\section{Informações do autor principal:}

Fernanda Britto de Melo Silva

Rua Ministro Viveiros de Castro, 82

Copacabana- Rio de Janeiro- RJ, Brasil.

CEP: 22021010

Email: fernandabrittodemelo2@hotmail.com

Telefone: +5521998177665 


\title{
Resumo
}

Sialolitos são estruturas calcificadas, que se desenvolvem no interior do sistema ductal salivar em decorrência da deposição de sais de cálcio ao redor de um acúmulo de restos orgânicos no lúmen do ducto glandular. Acometem mais frequentemente a glândula submandibular e são a causa mais comum de inflamações agudas ou crônicas nas glândulas salivares maiores. São mais frequentes em pacientes de 30 a 40 anos e duas vezes mais em homens do que em mulheres. Os sintomas geralmente se apresentam com o aumento da glândula salivar gerando tumefação local, febre, disfagia e dor. O diagnóstico correto envolve exame clínico, inspeção, palpação, manipulação da glândula e exames radiográficos. Podem ser evidenciados por radiografias convencionais, tomografia computadorizada, ressonância magnética, ultrassonografia, cintilografia, sialoendoscopia e sialografia. O tratamento inclui a eliminação espontânea mediante orientações ou uso de medicamentos sialogogos, ou a remoção cirúrgica do sialolito, sendo necessária, em alguns casos, a exérese da própria glândula. Este trabalho tem como objetivo relatar o caso clínico do paciente G.L.C, 70 anos de idade, sexo masculino, que compareceu ao Serviço de Cirurgia Oral e Maxilofacial do Hospital Federal dos Servidores do Estado, com queixa principal de dor e aumento de volume. Apresentava laudo de ultrassonografia evidenciando a presença de 3 cálculos medindo $10 \mathrm{~mm}, 9 \mathrm{~mm}$ e $8 \mathrm{~mm}$ em glândula Submandibular direita. $\mathrm{O}$ paciente foi submetido à procedimento cirúrgico sob anestesia geral para exérese da glândula Submandibular que correu sem intercorrências. O paciente segue em acompanhamento pós operatório de 1 ano com boa evolução e sem sintomatologia.

Palavras-chave: sialolito; glândula submandibular; cirurgia oral e maxilofacial.

\begin{abstract}
Sialolites are calcified structures that develop within the salivary duct system due to the deposition of calcium salts around an accumulation of organic debris in the lumen of the glandular duct. They most often affect the Submandibular gland and are the most common cause of acute or chronic inflammation in the larger salivary glands. They are more


common in patients aged 30 to 40 years and twice as often in men than in women. Symptoms usually present with enlargement of the salivary gland leading to local swelling, fever, dysphagia and pain. The correct diagnosis involves clinical examination, inspection, palpation, manipulation of the gland and radiographic examinations. They can be evidenced by conventional radiographs, computed tomography, magnetic resonance imaging, ultrasound, scintigraphy, sialendoscopy and sialography. Treatment includes spontaneous elimination through guidance or use of sialogogues, or surgical removal of the sialolith, and in some cases the removal of the gland itself is necessary. This paper aims to report the clinical case of patient G.L.C, 70 years old, male, who attended the Oral and Maxillofacial Surgery Service of the Federal Hospital of the State Servants, with the main complaint of pain and swelling. He presented ultrasound report showing the presence of 3 calculi measuring $10 \mathrm{~mm}, 9 \mathrm{~mm}$ and $8 \mathrm{~mm}$ in the right Submandibular gland. The patient underwent a surgical procedure under general anesthesia for submandibular gland excision that ran uneventfully. The patient follows a 1-year postoperative followup with good evolution and no symptoms

Keywords: sialolite; submandibular gland; oral and maxillofacial surgery. 


\section{Introdução}

Sialolitos são estruturas mineralizadas que se desenvolvem no interior dos ductos das glândulas salivares, podendo ser encontrados no seu interior ou ao longo do ducto. A sialolitíase tem etiologia incerta, que pode estar relacionada à existência de sialodenite crônica e não tem correlação com alterações sistêmicas no metabolismo de cálcio e fósforo (NEVILLE BW, DAMM DD. et al. 2009).

Aproximadamente $80 \%$ dos sialolitos acometem a glândula submandibular. Destes, cerca de $40 \%$ estão localizados na porção distal do ducto (NEVILLE BW, DAMM DD. et al. 2009; COMBES J, KARAVIDAS K. et al. 2009; Zhao YN, Zhang YQ. et al. 2019). O maior envolvimento desta glândula salivar pode estar associado ao trajeto ascendente e tortuoso do ducto de Wharton e a produção de saliva mais espessa que as demais glândulas (EPKER BN, 1972). Embora possa se desenvolver em pacientes de qualquer idade, a sialolitíase tem maior incidência em adultos jovens (NEVILLE BW, DAMM DD. et al. 2009). O tamanho dos cálculos salivares é variável, podendo atingir alguns centímetros. No entanto, a maioria apresenta dimensões inferiores a $10 \mathrm{~mm}$ (BODNER, L. 2002; LUSTMANN J, REGEV E. et al., 1990).

As manifestações clínicas da sialolitíase de glândulas salivares maiores incluem dor e/ou de aumento de volume da glândula afetada, comumente exacerbados pela alimentação. A intensidade dos sintomas é influenciada pelo grau de obstrução ao fluxo salivar exercido pelo sialolito. Sialodenite aguda ou crônica pode se desenvolver em decorrência da obstrução ductal (NEVILLE BW, DAMM DD. et al. 2009; COMBES J, KARAVIDAS K. et al. 2009; BODNER, L. 2002).

O grau variável de mineralização dos sialolitos faz com que nem todas as lesões sejam visíveis em radiografias convencionais. Entretanto, estas frequentemente se apresentam como uma massa radiopaca. Dependendo da localização, radiografias oclusais, periapicais e panorâmicas podem permitir sua visualização. Além destes 
exames, ultrassonografia e a tomografia computadorizada podem ser ferramentas úteis (NEVILLE BW, DAMM DD. et al. 2009; COMBES J, KARAVIDAS K. et al. 2009; EPKER BN, 1972).

A escolha do tratamento é depende do tamanho e da localização das lesões. Sialolitos pequenos podem ser tratados com a mobilização da estrutura calcificada em direção ao orifício de saída do ducto. Para tal, são adotadas medidas como massagens, aplicação de calor local e uso de sialogogos. Outras opções conservadoras são a litotripsia de ondas de choque extracorpórea e a sialoendoscopia intervencional. Cálculos na região distal do ducto de Warthon podem ser removidos através de acesso intraoral. Em contrapartida, aqueles localizados no parênquima glandular ou ducto proximal comumente demandam sialadenectomia via acesso extraoral (NEVILLE BW, DAMM DD. et al. 2009; COMBES J, KARAVIDAS K. et al. 2009).

\section{Considerações éticas}

Os procedimentos empregados neste trabalho seguiram os padrões éticos propostos pela Declaração de Helsinque de 1975, revisada em 2000. O paciente foi tratado na Faculdade de Odontologia da Universidade Federal Fluminense através da Clínica de dentes inclusos com os termos, de consentimento livre e esclarecido e de uso de imagens e dados para publicação científica, assinados.

O estudo foi escrito seguindo as Diretrizes do Relatório de Caso Clínico (CARE, www.care-statement.org).

\section{Relato de Caso}

Paciente G.L.C., leucoderma, sexo masculino, 70 anos de idade, procurou o Serviço de Cirurgia Oral e Maxilofacial do Hospital Federal dos Servidores do Estado, com queixa principal de dor e aumento de volume em região submandibular do lado direito.

Ao exame clínico o paciente apresentava assimetria facial em consequência do aumento de volume de consistência firme, móvel e com dor à palpação em região de 
Glândula Submandibular do lado direito (Figura 1A e 1B). No exame intraoral, não apresentava alterações de normalidade em ordenha da glândula.

No exame de imagem, apresentava laudo de ultrassonografia evidenciando a presença de 3 cálculos medindo 10mm, 9mm e $8 \mathrm{~mm}$ em glândula Submandibular direita. $\mathrm{Na}$ radiografia panorâmica de face era possível evidenciar imagem radiopaca de grandes proporções em região compatível com a Glândula Submandibular sugestiva de Sialolito (Figura 1C). 
Figura 1. A) Vista lateral evidenciando aumento de volume; B) Visão ínfero-

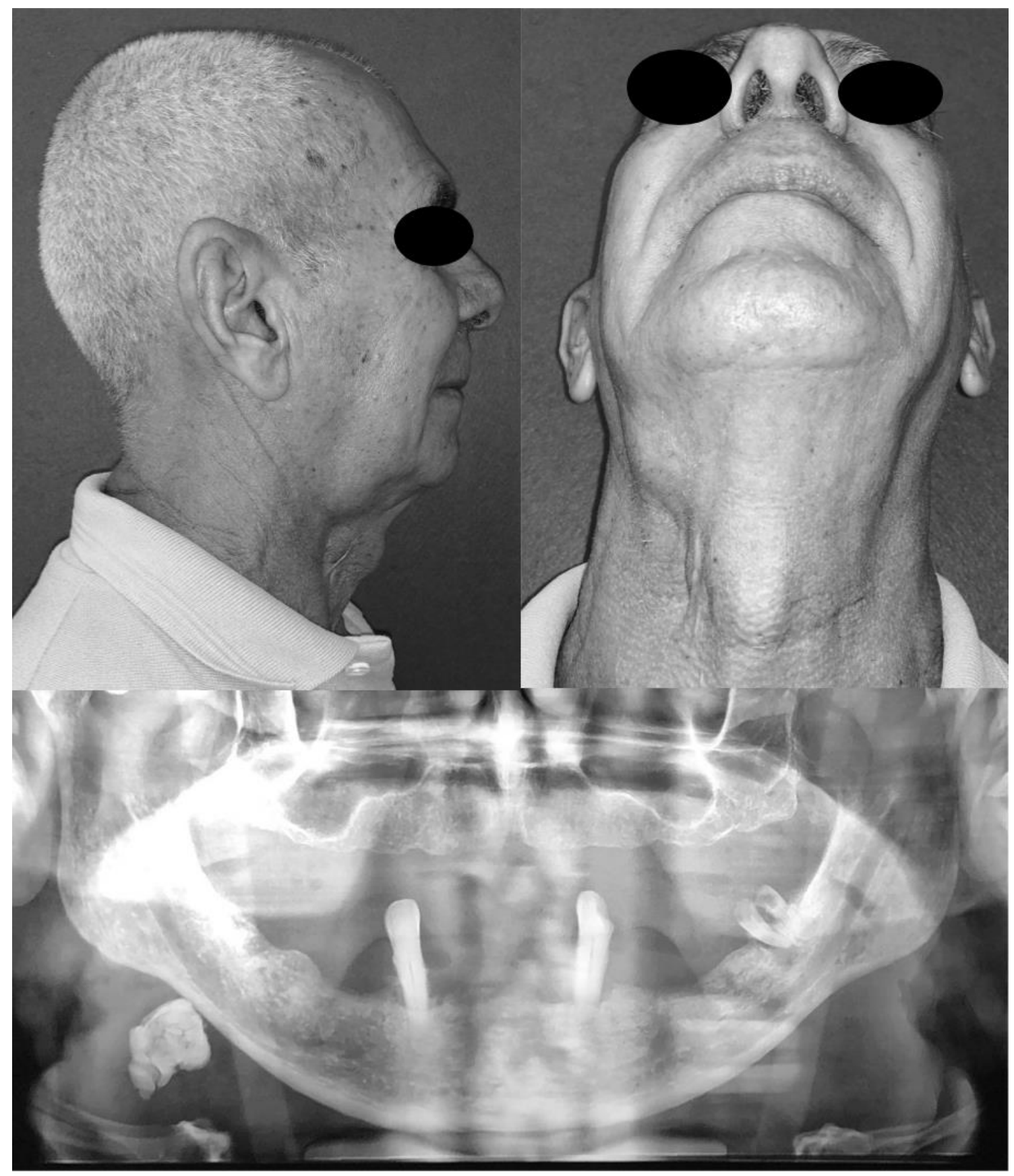

superior evidenciando assimetria; C) Radiografia Panorâmica de face apresentando imagem radiopaca em região de Glândula Submandibular. 
O tratamento proposto foi de exérese da Glândula Submandibular através de acesso Submandibular em ambiente de Centro Cirúrgico.

O procedimento cirúrgico foi realizado sob anestesia geral e intubação nasotraqueal. Após seu posicionamento e a colocação dos campos estéreis, foi realizada marcação das linhas de incisão para o acesso submandibular do lado direito e infiltração com lidocaína 2\% e epinefrina 1:100.000 para anestesia e vasoconstricção locaais. Após realizada a incisão de pele e subcutâneo, foi feita a divulsão dos tecidos para uma melhor elasticidade e exposição, seguida de incisão do músculo Platisma. Seguindo a divulsão com profundidade até o plano da camada superficial da fáscia cervical profunda e através de dissecção, ligadura da Artéria e Veia Faciais, e do ducto da glândula Submandibular, a mesma foi ressecada em sua totalidade (Figura 2A), contendo os sialolitos em seu interior (Figura 2B). O procedimento correu sem intercorrências cirúrgicas, com bom controle hemostático e o material foi encaminhado para o exame histopatológico.

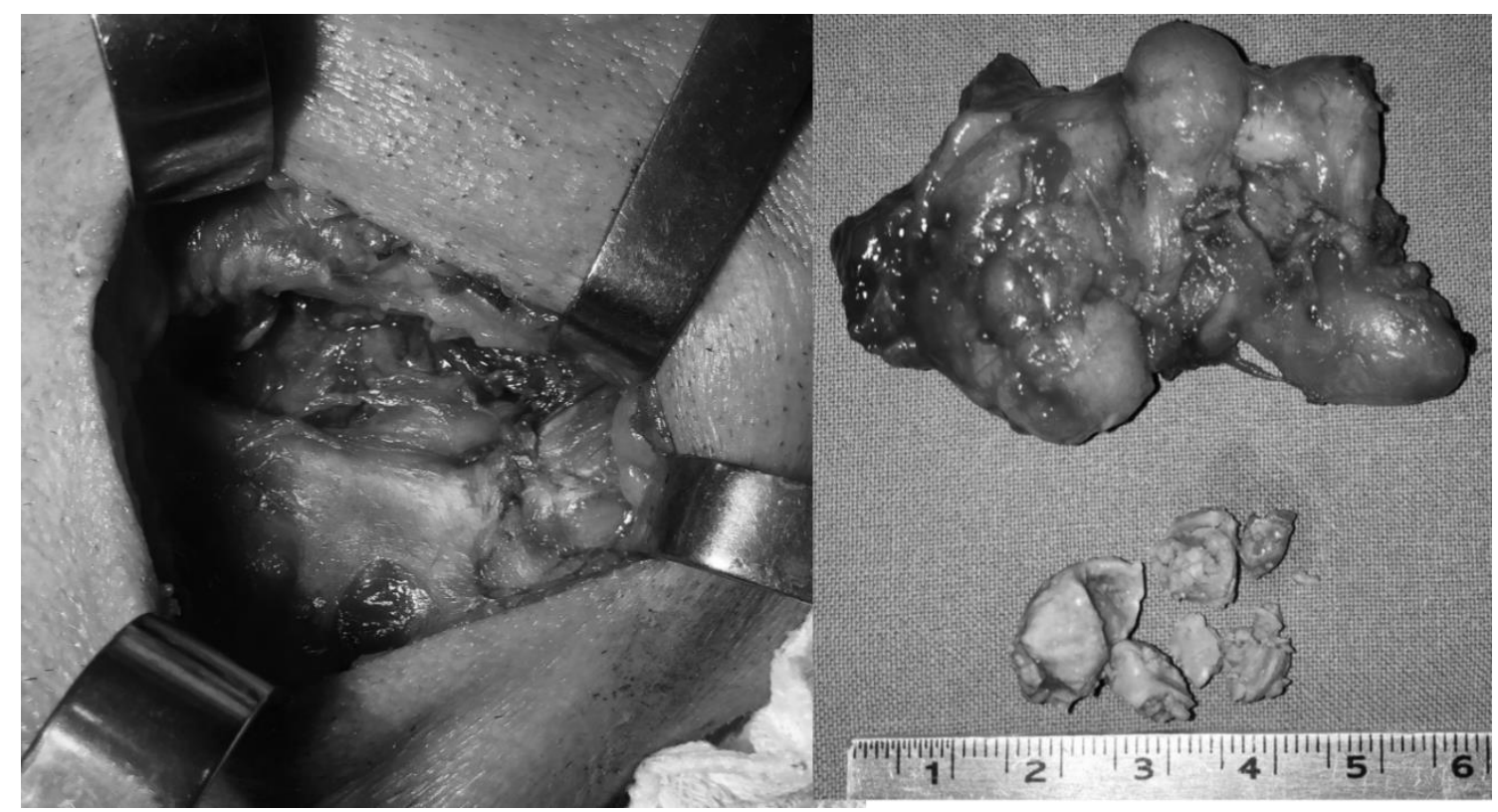

Figura 2. A) Acesso cirúrgico Submandibular evidenciando o plano cirúrgico após remoção da glândula. B) Exérese total da Glândula Submandibular e os cálculos removidos do seu interior. 
Foi realizada síntese tecidual e o fechamento por planos foi respeitado, utilizandose fio Vicryl 3.0 para as camadas musculares e de subcutâneo e fio Nylon 5.0 para fechamento de pele.

Durante o acompanhamento pós-operatório imediato, o paciente apresentou boa evolução, sem complicações na ferida cirúrgica e com resolução das queixas prévias. Além disso, o paciente não apresenta sinais de injúria ao N. Marginal da Mandíbula nos teste de movimento do lábio (Figura 3A e 3B).

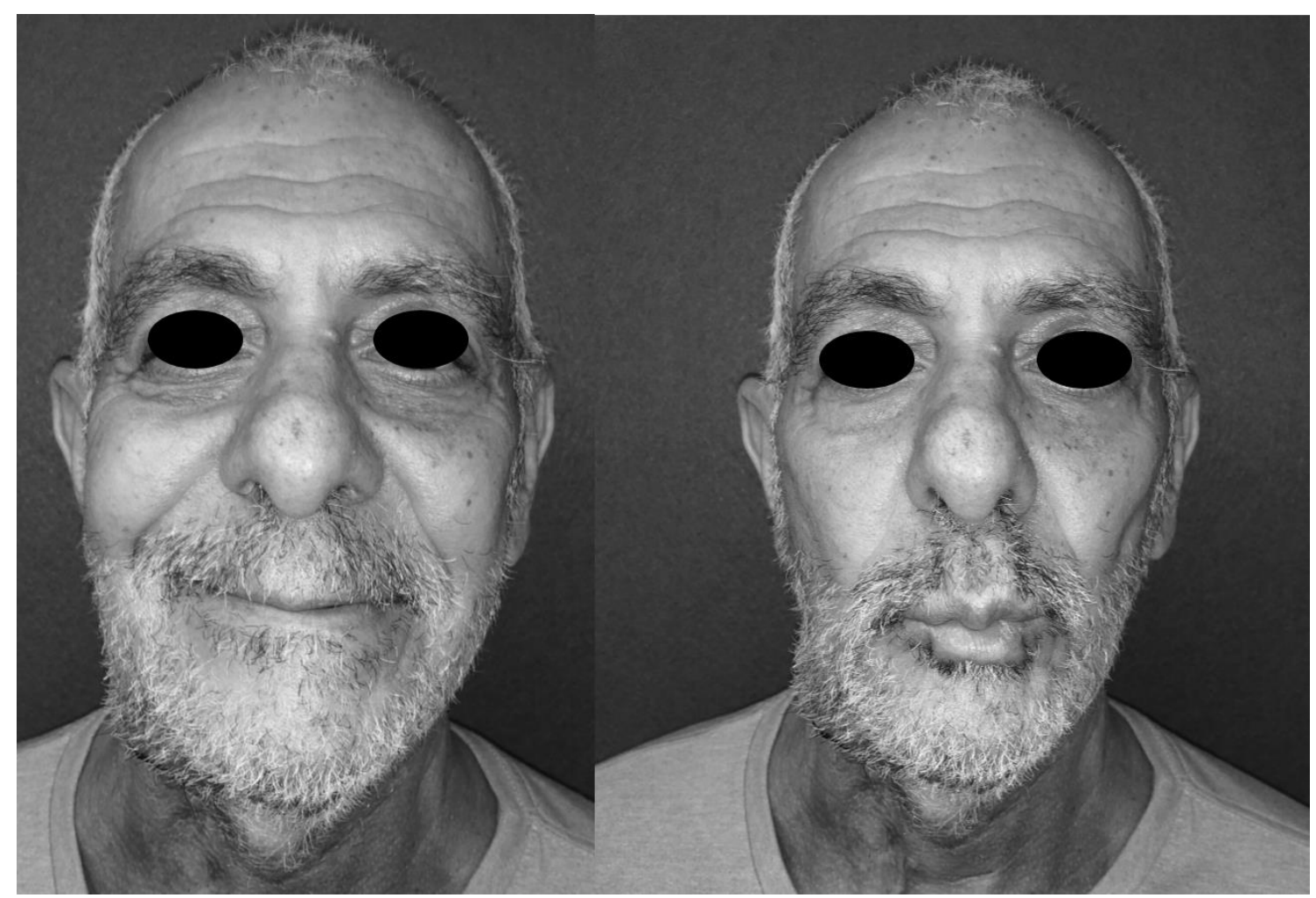

Figura 3. A) Teste do Sorriso forte evidenciando N. Marginal da Mandíbula preservado; B) Preservação do N. Marginal da Mandíbula no movimento da musculatura do lábio.

Em acompanhamento de 1 mês, ao exame clínico o paciente segue sem alterações ou sintomatologia. O laudo do exame histopatológico foi compatível com sialolitíase com sialodenite crônica difusa.

\section{Discussão}


A maioria dos casos de sialolitíase se apresenta através da formação de uma única estrutura mineralizada isolada (NEVILLE BW, DAMM DD. et al. 2009). O caso apresentado mostra a formação de três sialolitos, passíveis de identificação já na etapa diagnóstica através de exame ultrassonográfico.

Dentre as possíveis abordagens terapêuticas dos sialolitos, a opção pela exérese da glândula submandibular foi baseada especialmente na localização intraglandular destes. Esta modalidade de tratamento é eficaz na resolução do quadro clínico, porém resulta em cicatriz aparente e pode ocasionar alterações motoras em face, em consequência do acesso cirúrgico. Portanto, esta opção deve ter uso restrito a lesões sintomáticas intraglandulares ou em ducto proximal(COMBES J, KARAVIDAS K. et al. 2009).

Técnicas minimamente invasivas, como a litotripsia extracorpórea por ondas de choque e a sialoendoscopia intervencional, permitem preservar remover a obstrução preservando o tecido glandular. Abordagens minimamente invasivas são justificadas pela retomada da função normal e assintomática da glândula salivar após a retirada do cálculo na maioria dos pacientes (COMBES J, KARAVIDAS K. et al. 2009; MAKDISSI J, ESCUDIER MP. et al., 2004). Contudo, a litotripsia extracorpórea por ondas de choque é última apenas para cálculos com dimensões inferiores a $8 \mathrm{~mm}$ e mostram eficácia na resolução dos sintomas em apenas aproximadamente um terço dos casos (COMBES J, KARAVIDAS K. et al. 2009; ZENK J, BOZZATO A. et al., 2004).

\section{Conclusão}

A exérese da glândula submandibular foi considerada eficaz e bem-sucedida, uma vez que objetivo de eliminar a sintomatologia foi alcançado e não houve lesão do nervo marginal da mandíbula, frequentemente relacionada ao acesso cirúrgico submandibular. Podemos concluir que, embora técnicas minimamente invasivas estejam disponíveis, a exérese da glândula submandibular permanece como uma modalidade terapêutica adequada para o tratamento de sialolitíase, em especial nos casos com múltiplos ou grande sialolitos. 


\section{Referências Bibliográficas}

1. Neville BW, Damm DD. et al. Patologia das glândulas salivares. In: Patologia oral \& Maxilofacial. 3 ed. Rio de Janeiro: Elsevier, 2009. Cap 11, p. 455-474.

2. Combes J, Karavidas K. et al. Intraoral removal of proximal submandibular stones - an alternative to sialadenectomy? Int J Oral Maxillofac Surg, 38(8): 813-816, ago 2009.

3. Zhao YN, Zhang YQ. et al. Treatment strategy of hilar and intraglandular stones in wharton's duct: A 12-year experience. Laryngoscope. 2019 Nov 6. doi: 10.1002/lary.28361 [Epub ahead of print].

4. Epker BN. Obstructive and inflammatory diseases of the major salivary glands Oral Surg Oral Med Oral Pathol. 33(1): 2-27, 1972.

5. Bodner, L. Giant salivary gland calculi: Diagnostic imaging and surgical management. Oral Surg Oral Med Oral Pathol Oral Radiol Endod, 94(3): 320-323, set 2002 .

6. Lustmann J, Regev E. et al. Sialolithiasis: A survey on 245 patients and a review of the literature. Int J Oral Maxillofac Surg. 19(3): 135-8, 1990.

7. Makdissi J, Escudier MP. et al. Glandular function after intraoral removal of salivary calculi from the hilum of the submandibular gland. Br J Oral Maxillofac Surg. 42(6): 538-41, 2004.

8. Zenk J, Bozzato A. et al. Extracorporeal shock wave lithotripsy of submandibular stones: evaluation after 10 years. Otol Rhinol Laryngol. 113(5): 378-83, 2004. 